\title{
Le travail des élèves au cœur des tensions de l'école
}

\section{Roger-François Gauthier}

\section{(2) OpenEdition}

Journals

Édition électronique

URL : https://journals.openedition.org/ries/1832

DOI : $10.4000 /$ ries. 1832

ISSN : 2261-4265

\section{Éditeur}

France Education international

\section{Édition imprimée}

Date de publication : 1 avril 2002

Pagination : 29-38

ISBN : 2-84520-553-8

ISSN : $1254-4590$

\section{Référence électronique}

Roger-François Gauthier, «Le travail des élèves au cœur des tensions de l'école », Revue internationale d'éducation de Sèvres [En ligne], 29 | avril 2002, mis en ligne le 24 novembre 2011, consulté le 06 juillet 2021. URL : http://journals.openedition.org/ries/1832; DOI : https://doi.org/10.4000/ries.1832

(C) Tous droits réservés 


\section{L'élève aujourd'hui : façons d'apprendre}

\section{Introduction}

\section{Le travail des élèves au cœur des tensions de l'école}

\section{Roger-François Gauthier}

Les lecteurs français de la Revue internationale d'éducation - Sèvres ne seront guère surpris du choix du thème de ce numéro, tant l'actualité éducative de ce pays fourmille d'initiatives officielles, souvent inspirées par les propositions antérieures de divers mouvements pédagogiques, qui s'attachent au champ du "travail des élèves ».

Si l'on veut bien y prêter attention, ce fait n'est d'ailleurs pas une petite surprise, car ce n'est que récemment que l'étrange silence réglementaire qui marquait depuis plus de quarante ans la définition du "travail de l'élève » (l'Inspection générale a observé que les derniers textes normatifs sur le sujet du travail personnel remontaient par exemple à 1961) a été brisé par un impressionnant concert de prescriptions ambitieuses: une réalité qui paraissait souvent l'oubliée, la non-dite, voire la refoulée des réformes successives est soudain consacrée pour "tirer » la réforme présente !

Philippe Joutard et Marc Baconnet nous rappellent précisément, à propos des réformes en cours au collège et au lycée, que nous assistons en fait, depuis peu d'années et sous des intitulés divers qui ont presque tous en commun le recours au mot de "travail», à la diffusion presque simultanée, dans le sens étonnant de l' "aval» des classes préparatoires aux grandes écoles vers l' " amont » de l'enseignement primaire, d'un modèle traduisant une unité, exceptionnelle dans l'histoire récente de l'éducation en France au-dessus du tronçonnement du système, de la stratégie et des objectifs officiels.

En tant que stratégie, tout se passe comme si les responsables nationaux considéraient de plus en plus souvent qu'agir sur le travail des élèves consistait à actionner un des leviers les plus puissants de transformation du système tout entier ; s'agissant des objectifs, développer l'autonomie intellectuelle de l'élève deviendrait l'une des finalités centrales du système d'éducation, sinon la finalité des finalités.

C’est donc le moment propice, semble-t-il, pour réfléchir, conformément aux ambitions de cette revue, sans enfermer son cerveau dans un hexagone, 
à ce «travail des élèves » que l'école française paraît en un sens redécouvrir et valoriser.

En quoi les évolutions qui ont cours en France rencontrent-elles l'écho d'autres systèmes scolaires? En quoi des débats, "étrangers » en apparence, peuvent-ils aider des acteurs d'autres pays à parfois mieux comprendre ce qu'ils font ? Comment le champ se structure-t-il, et entre quels écueils et garde-fous d'autres avancent-ils?

D'ailleurs, quel que soit le « souverainisme » des systèmes d'éducation, et leur partie liée avec les spécificités nationales, la question du «travail de l'élève » est d'autant plus intéressante à observer sous un angle "international » qu'elle paraît bien aujourd'hui transcender quelques frontières: quels pays développés, par exemple, ne sont pas en effet conduits par la tension qui existe entre l'objectif démocratique d'ouverture de l'école à tous et le rejet, par une partie des élèves au moins, des modèles scolaires proposés, à se poser la question des activités que cette école propose aux élèves? Quels ne perçoivent pas que ce que font les élèves en classe n'est désormais en bien des cas qu'une partie d'un ensemble d'activités que leur dicte, souvent par l'intermédiaire de leurs familles, la compétition scolaire la plus acharnée? Quels ne comprennent pas que, sans qu'il ait certes à dicter sa loi à l'école, l'environnement documentaire et culturel, largement façonné par les techniques et réseaux contemporains, dans lequel évoluent les élèves en dehors même de l'école, change bien des choses au royaume de l'enseignement simultané traditionnel (ce qu'illustre ici l'expérimentation par les TICE, en cours dans quatre Länder allemands, relatée par Sandrine Lamer)?

Les auteurs des différents articles ont traité le sujet des points de vue différents qui étaient les leurs, et ont le grand mérite de l'avoir fait avec sincérité et honnêteté : aucun article ne prétend avoir découvert «le » modèle qui devrait s'imposer, tous nous disent leurs espoirs, leurs interrogations ou leurs doutes, et ceux qui sont les plus avancés sur la voie de l'autonomie du travail de l'élève, comme sans doute les Néerlandais, peuvent utilement mettre les autres en garde contre certaines dérives ou effets non désirés de telle innovation pédagogique. En d'autres cas l'étude d'une situation étrangère a le seul, mais apprécié, mérite de nous rendre un peu moins naïfs en nous permettant de brûler quelques épisodes de romans pédagogiques qui souvent se ressemblent, ou en se bornant à nous présenter de façon simultanée une palette de possibles que l'histoire ailleurs dévoile successivement!

Avant d'écouter ces voix venues d'ailleurs, ne manquons pas de rappeler en quoi se joue actuellement en France, sur ce sujet du «travail» de l'élève, une petite révolution : les innovations que l'institution - même en France - entend instituer aux différents niveaux de l'enseignement, pourraient bien mériter ce terme, comme le montre ici Marc Baconnet à propos des «travaux personnels encadrés ». 
En quoi ? Philippe Joutard le dit bien, quand il précise que sont essentiels désormais pour les élèves à la fois la maîtrise d'une recherche documentaire et la capacité à " produire », pas nécessairement un chef d'œuvre, mais le résultat d'un «travail », comme c'est depuis toujours le cas dans l'enseignement professionnel.

Révolution ne serait-ce que parce que le «travail » demandé aux élèves sort ainsi d'une clandestinité pluriséculaire de l'enseignement classique pour être "défini » en tant que tel, et parce que l'impact attendu, à savoir que les élèves réussissent mieux leurs apprentissages et surmontent plus souvent, en acquerrant davantage d'autonomie, les fatalismes socio-scolaires, est révolutionnaire.

Il ne faut en effet pas négliger de rappeler en quoi le " travail scolaire » est traditionnellement un quasi-clandestin.

\section{UN MANQUE ANCIEN DE CADRAGE PHILOSOPHIQUE}

La philosophie nous dit peu de choses sur le «travail» de celui qui apprend, non seulement parce que l'école serait le lieu de l'éternel «loisir», conformément à son étymologie, mais surtout parce que l'école occidentale est fondée sur une philosophie de la connaissance, empruntée à Platon, qui continue de faire de l'enseignement un "dévoilement», et pour laquelle l'apprentissage ne nécessite guère au fond que le temps qu'il faut pour se rendre à l' «évidence ». À cet égard, l'antique fonction de la parole dans cette archéologie scolaire est en Italie particulièrement remarquable.

Bien sûr l'école d'autrefois imposait de nombreuses heures d' "étude ", beaucoup plus que l'école d'aujourd'hui, davantage prodigue en " cours ", mais ces études consistaient en entraînements divers censés faciliter l'accès à la vérité, elles n'étaient jamais à proprement parler un «travail» au sens où l'entend par exemple un apprentissage professionnel.

Quant au temps de l'enseignement proprement dit, il est bien souvent le lieu d'un «cours dialogué » qui, même quand il n'est pas l'étonnante interrogation de ceux qui ignorent par celui qui sait, n'en dessine pas moins pour l'élève une activité dont on perçoit qu'elle est hétérogène par rapport à ce qu'on nomme ordinairement «travail».

\section{UN MANQUE DE SIGNIFICATION SOCIALE EXTERNE}

Il y a sans doute en français dans l'expression «passe ton bac d'abord! » une analyse plus fine qu'il n'y paraît du fonctionnement réel de l'école : l'école ne "vaut» que comme tension vers le moment de l'obtention du diplôme, mais ce qu'elle fait au quotidien n'a pas en soi de «valeur». 
Sans reconnaissance externe, les activités scolaires oscillent entre des approximations mimétiques contestables du «travail » véritable, comme quand le «labo de sciences" est trop légèrement rapproché du laboratoire de recherches ou l'atelier de technologie de l'atelier professionnel et des exercices dont le formalisme typiquement scolaire (la dictée, comme l'eût fait jadis le thème latin, fournit en France un exemple facile) fera dans le souvenir monter la vie durant des larmes d'attendrissement ou de ressentiment.

\section{UN MANQUE DE CADRAGE PÉDAGOGIQUE}

Comment ne pas s'étonner en effet de constater qu'alors que les horaires d'enseignement des différentes disciplines sont réglés au quart d'heure près au plan national à la suite d'infinis marchandages, le flou le plus complet règne en ce qui concerne le temps prescrit par les professeurs de ces mêmes disciplines pour le travail des élèves en dehors du cadre de la classe ?

Les inspecteurs généraux français qui ont eu à enquêter dans des établissements secondaires à propos du travail personnel des élèves relatent la surprise intéressée des professeurs interrogés dans le cadre de ces enquêtes quand il leur est donné de découvrir la diversité des pratiques de leurs collègues : on sait aussi aujourd'hui qu'en certaines classes secondaires les professeurs ne donnent plus de travail en dehors de la classe, pour le motif qu'il ne serait pas fait ou qu'il vérifierait l'aptitude des parents plutôt que celle des élèves, qu'en d'autres classes le travail personnel, pour des motifs similaires, n'est pas "noté », et des élèves interrogés sur le temps qu'ils croient devoir passer à ces travaux personnels font, alors même qu'ils appartiennent à la même classe, des déclarations d'une invraisemblable disparité.

C'est précisément là le paradoxe de la situation française : comment un objet si peu assuré sur ses bases que le travail scolaire lui-même pourrait-il être le levier par lequel adviendraient des changements significatifs à l'intérieur de l'école? Il faut en effet se souvenir qu'il s'agit d'un domaine où les habitudes des enseignants ainsi que la pression des familles sont traditionnellement plus forts que les injonctions officielles, comme le montre l'inanité des nombreux textes ayant prétendu dans le passé interdire les devoirs à l'école primaire ou organiser ceux de sixième!

Il y a donc, sur le chemin de cette renaissance de l'attention portée au travail des élèves, bien des questions posées. Nous en privilégierons ici trois :

\section{LE RÔLE DU POUVOIR CENTRAL ET SON MODE DE MANAGEMENT}

Mariano Palamidessi et Daniel Galarza nous le disent bien : dans la phase où un système éducatif se construit, en référence par exemple à un État national, il a souvent besoin de définir de façon homogène et centralisée les 
méthodes pédagogiques en vigueur et la «tâche » des élèves est alors prescrite en un sens qui laisse peu de place aux variations ou à l'autonomie.

Dans les pays en revanche dont la politique éducative met en avant un objectif de décentralisation, en particulier au niveau de l'établissement scolaire, ce dont la situation italienne contemporaine décryptée ici par Teresa Longo fournit un bon exemple, on peut s'attendre à ce que la norme sur les activités des élèves devienne moins stricte, voire s'efface devant une définition locale de ces travaux, inspirée par les besoins particuliers du public de chaque établissement, le contexte d'action de cet établissement, son projet, etc.

L'affaire n'est toutefois pas simple si au même moment, et c'est la situation française, l'institution centrale intervient fortement en un terrain où elle ne s'aventurait guère jusque là et prescrit une nouvelle donne pour les "travaux d'élèves» aux différents niveaux scolaires : la tension existe et se manifestera probablement en un certain nombre de cas entre l' «autonomie » de l'établissement et la prescription nationale mettant au cœur des préoccupations de l'école le développement de l'autonomie... de l'élève.

On peut toutefois raisonnablement penser que la plupart du temps, l'injonction faite aux établissements d'innover par les "parcours de découverte », les «travaux personnels encadrés» ou encore « les projets pluridisciplinaires à composante professionnelle » viendra donner de la chair au projet d'établissement plutôt qu'elle n'entrera en conflit avec ses dispositions : l'autonomie de l'établissement n'est pas en effet un objectif en soi, c'est pour lui le moyen de mieux répondre aux besoins de ses élèves et de favoriser leurs apprentissages dans un contexte déterminé, propos qui est en totale conformité avec les ambitions des "nouveaux travaux».

Il est clair en revanche qu'en intervenant en ce sens sur le «travail des élèves ", le pouvoir politique joue pleinement son rôle (et Teresa Longo nous montre en quoi ce qui était demandé aux élèves a varié, au vingtième siècle, en Italie, selon qui était au pouvoir à Rome !), puisqu'il doit veiller de par la loi à promouvoir une plus grande égalité des élèves devant l'accès au savoir : on sait à quel point les différences sociales sont discriminantes pour ce qui concerne l'exercice du "métier d'élève ", la conquête de l'autonomie et la construction de la synthèse des savoirs.

Les élèves « en difficulté » sont en effet souvent ceux qui ne parviennent pas à lever le nez au-dessus du caléidoscope des différentes «tâches » scolaires qui leur sont proposées et des années d'études successives, même s’ils parviennent à « réussir » des tâches «taylorisées » et à « passer » dans la classe supérieure.

Il y a au fond de ces positions politiques l'idée que c'est peut-être devant leur propre travail que les élèves sont le plus "inégaux », ce que l'article argentin met bien en valeur, quand l'école les laisse se débrouiller avec des consignes floues, un implicite culturel paralysant, ou de pauvres outils documentaires, sans même parler de l'aide familiale par définition inégalitaire : 
en s'attaquant au "travail de l'élève », c'est à cet ensemble de fatalités que l'école s'attaque. "Enfin! », aurait-on envie de rajouter !

Mais en relisant alors l'article sur la situation aux Pays-Bas de Sarah Blom et Chantal Weststrate, qui nous rappelle dans ses interrogations mêmes que l'autonomie peut être insupportablement synonyme d'élitisme social si on oublie qu'elle est une conquête, et en rien une donnée, peut-être la conquête la plus éminente, la plus difficile, celle en tout cas qui rend totalement indispensables l'école, le maître, et tout le regard "en synthèse » dont il est capable sur les apprentissages en train de se faire.

\section{INTERACTIONS \\ ENTRE TRAVAIL SCOLAIRE ET DEMANDES SOCIALES}

C'est bien en effet la spécificité du travail scolaire : défini à l'intérieur du cadre de l'école par les maîtres qui le prescrivent à leurs élèves, il consiste souvent en des activités à faire "à la maison ", et se trouve ainsi être l'interface la plus visible et la plus lisible entre l'école et la famille, quel que soit le sort qui lui est réservé après le temps scolaire.

Or qu'il s'agisse de la pression de la compétition scolaire et sociale, aussi bien que des sollicitations innombrables des enfants et des adolescents par des « activités " extra-scolaires, tout montre que l'école n'a pas en tous les cas la main.

Si elle entend ne plus laisser le travail des élèves dans le flou, dans l'extrême disparité des situations et des exigences, et dans la position symbolique secondaire qui est bien souvent la sienne, actuellement au moins, en France, elle doit être consciente de l'importance de la clarté du message à faire passer aux familles, à la société, et à tous ses partenaires, comme les collectivités territoriales.

Les projets d'établissements comme les conseils d'administration doivent inscrire les nouvelles modalités de travail des élèves au centre de leur ordre du jour, étant entendu que rien ne réussira en la matière sans une reconnaissance du milieu et des familles qui n'est pas acquise. En Italie, ces matières ont pu progressivement s'ouvrir à une approche véritablement plurielle au sein de l'établissement, impliquant les élèves, leurs familles, les enseignants, la direction...

S'il s'agit bien de la part de l'école d'une reprise de parole, dotée d'un sens politique, elle ne pourra laisser dans l'ombre des questions comme le sens externe des exercices prescrits, comme le volume du travail demandé, mesuré en temps et rapporté au temps du travail des adultes, comme la nature des aides dont l'élève peut bénéficier, selon que l'école doit être en tous les cas son propre recours ou qu'il convient de faire appel à une aide associative ou privée, comme l'outillage dont il devra bénéficier, dans l'établissement et dans sa famille (à cet égard, notre article sur l'école allemande fait état d'une préoccupation particulière pour l'outillage de l'élève), comme le rapport entre ce travail scolaire et les évaluations externes de ce travail, des bulletins de notes aux résultats aux 
examens. Les Néerlandais ont bien compris l'intérêt d'une démarche de système en la matière, et qui a suivi l'actualité française sait bien que c'est précisément quand s'est posée la question de la prise en compte des TPE au baccalauréat que le dossier s'est tendu.

L'école rencontre bien sûr des résistances externes chaque fois qu'elle reprend le flambeau de l'utopie, dans un contexte consumériste qui ne lui demande souvent que la distinction sociale et la recette pour gagner : le projet que chacun puisse construire un savoir critique en affrontant, par des «travaux » personnels transcendant l'uniformité scolaire, la complexité authentique du monde n'ira jamais de soi car il est à contre-courant, pour des motifs différents, aussi bien de vigoureuses traditions pédagogiques que des tendances qui ont le soutien du marché ; il nécessitera de toute évidence un accord politique sur ce qui est peut-être le plus délicat, à savoir le statut social de l'apprentissage et la philosophie de la connaissance.

\section{L'ÉVOLUTION DE LA CONCEPTION DES ÉTUdES}

Personne n'est dupe, bien sûr : le changement dans la conception du travail des élèves vaut au-delà du travail des élèves; les illustrations françaises aussi bien qu'étrangères nous montrent que tout est lié.

Les conceptions linéaires des programmes d'enseignement, " en extension ", comme dit Philippe Joutard, ne sont pas les plus propices au travail des élèves puisqu'elles débouchent d'abord sur des prescriptions pour le travail... des professeurs.

L'expérience menée en Allemagne actuellement, SEMIK, propose un mode de gestion des programmes d'enseignement, recourant à une polyrythmie entre des phases de survol et des moments d'approfondissement, qui sont ceux du «travail » pour les élèves; elle peut donner à réfléchir ailleurs qu'en Allemagne.

L'alternance survol/approfondissement suppose à son tour l'élaboration de modalités adéquates de décision entre ce qui sera vu « dans les grandes lignes " et ce qui sera l'objet d'un véritable travail d'approfondissement: le programme devient un sujet sur lequel la parole de l'élève cesse d'être illégitime et qui appelle la négociation : l'illustration allemande est à cet égard intéressante.

Il est évident aussi que le caléidoscope disciplinaire sera qu'on le veuille ou non interpellé quand les élèves, dans le cadre de leurs travaux scolaires, prendront l'habitude de surmonter les frontières, de rechercher la synthèse et les voies du réinvestissement.

Il est tout aussi probable que, face à des programmes suivis de manière moins linéaire et plus diversifiée, l'élève (mais peut-être aussi ses enseignants successifs, comme ses évaluateurs) aura besoin de davantage de traces, de 
supports et de repères nouveaux dans sa scolarité : les "itinéraires » dont il est question dans l'article néerlandais, comme les «lacunes à combler» dont sont tenus responsables les lycéens italiens, ou encore la notion de Wissensmanagement proposée par Sandrine Lamer nous invitent à cette réflexion en nous fournissant quelques modèles.

L'organisation des classes, celle des emplois du temps, celle des lieux et ressources de la documentation, devront de leur côté se plier aux exigences diversifiées du travail des élèves, et cela créera de nombreuses tensions, chaque fois que jusqu'ici les exigences qui prévalaient étaient celles des prescriptions des programmes et de la gestion des groupes-classes. Il n'est pourtant pas envisageable que les conditions du travail des élèves ne facilitent pas pour eux la découverte des modalités ordinaires du travail hors de l'école et en particulier du travail collectif, ou en équipe.

La réflexion des enseignants sur leur propre métier est bien sûr impliquée par les évolutions du travail de leurs élèves, et l'article sur l'expérimentation allemande SEMIK nous le montre bien : que les travaux des élèves passent à l'explicite (voit-on tout le flou implicite qui entoure la simple consigne d' "apprendre une leçon»?), que les programmes d'enseignement soient l'occasion d'une parole, d'une réflexion et d'une stratégie elle aussi explicitée, que l'école ose le franchissement des frontières entre disciplines, que l'élève " conduise » effectivement un travail en autonomie, et c'est bien la figure de l'enseignant qui se distingue peu à peu, dans la conscience de ceux qui apprennent, de la figure du savoir. Libération? Sans doute, mais aussi, et souvent d'abord, déchirement.

D'autant qu'en un certain nombre de pays, et le rappel concernant ici les Pays-Bas est important, la promotion de méthodes pédagogiques demandant plus régulièrement à l'élève une production autonome s'est faite dans un climat de raréfaction de la ressource enseignante ou d'économies budgétaires qui a pu facilement laisser croire que l'objectif était de se passer des professeurs! Le sujet est en tous cas complexe et c'est le mérite du programme allemand SEMIK exposé ici de ne pas refuser a priori des questions délicates comme l'implication du développement du travail autonome sur l'évolution du volume horaire des « cours » proprement dits.

On sait d'ailleurs qu'en dehors même de telles menaces ou fantasmes, il peut y avoir de la part de certains enseignants une réticence à ce que ces nouveaux travaux, même quand ils n'en nient pas l'intérêt, empiètent sur le temps et les traditions de leur discipline, mais on sait aussi qu'il est urgent d'en finir avec la pédagogie des juxtapositions, pour les élèves, entre des temps et des préoccupations qui, quelque excellentes qu'elles soient, ne font que renforcer un peu plus, pour les plus en difficulté, la confusion du paysage.

On a assisté trop souvent à de véritables cours de méthodologie, juxtaposés à des enseignements disciplinaires qui n'en avaient cure, à des études 
dirigées qui, par un raccordement trop aléatoire aux besoins immédiats des élèves dans les disciplines, leur compliquaient encore un peu plus la vie, pour ne pas redouter que l'inauguration de ces "nouveaux travaux » débouche plus souvent sur l'adjonction d'une pièce nouvelle à un édifice déjà complexe que sur la reconsidération de l'ensemble.

Or l'enjeu est de taille : il en va peut-être de l'avenir de l'école qu'elle soit capable ou non d'unir ses voix et de surmonter ses clivages sur une conception de l'enseignement où soit privilégié l'investissement personnel de l'élève dans des travaux véritablement créateurs : Noëlle Sorin, du Québec, nous rappelle que l'exigence à respecter est bien celle de la culture et que si l'enseignement peut jouer, pour entraîner les élèves, de toutes les ressources de sa palette, y compris de celles qu'il doit au behaviourisme, c'est sans oublier que la fin est l'autonomie d'une personne et non la performance d'un animal dressé.

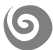

On voit bien en effet que la recherche de formes nouvelles d'activités des élèves, les impliquant davantage, supposant à la fois et leur demandant de construire un rapport plus personnel avec le savoir et avec le monde est commune à beaucoup de systèmes éducatifs, et peut-être un de ces étonnants points de rencontre des différents systèmes au-dessus de leur souverainisme premier.

On constate aussi qu'existe une tendance générale de l'école, renforcée par la perception quelque peu fantasmée du contexte de mondialisation des marchés, à ne pas rester sourde à une demande, que les classes économiquement favorisées ont formulée les premières, d'un enseignement qui cesserait de protéger les élèves, au moins en intention, contre les hypostases les plus cruelles de la compétition économique et sociale pour le pouvoir et l'argent : "privatisation ", " autonomie scolaire ", " offre de formations rares », " aide au travail » ou «services après la classe » font partie de la panoplie que la société de la compétition oppose avec vigueur et insidiation à l'ancienne utopie de l'école.

On peut craindre dans ce contexte que les innovations relatives au «travail » des élèves, si la volonté politique de les conforter dans un sens démocratique venait à manquer, deviennent de nouvelles caractéristiques socialement discriminantes entre les établissements ou entre les classes, soit que ces "nouveaux travaux», perspective qui n'est pas totalement à écarter, soient abandonnés à quelque enlisement ou marginalité et deviennent des activités " pour élèves en difficulté », pendant que "les autres » continueraient à "faire des choses sérieuses ", soit qu'ils soient un succès et améliorent effectivement la formation des élèves, mais en profitant d'abord à ceux qui étaient déjà dans la position la plus favorable. 
On évitera d'autant mieux ces dévoiements que tous les décideurs et acteurs des systèmes situeront bien l'ensemble des questions relatives au "travail des élèves" ailleurs que dans les marges implicites de leur propre action.

Quant au «travail » des élèves, l'école a sans doute raison non seulement de ne plus le nier, mais aussi de le susciter et de lui donner matière à s'illustrer, mais il sera sans doute important d'inciter les élèves à réfléchir mieux à sa signification : il n'est pas assimilable par nature au travail de leurs parents ni des adultes qui les entourent en dehors de l'école, il ne l'est pas au travail de leurs professeurs, (et à cet égard il conviendrait de surmonter une étrange mais vivace opposition entre la classe qui serait centrée sur le travail du maitre et l'apprentissage sur celui de l'élève !...), et pourtant il doit se situer parmi eux, en référence progressive au travail des hommes et à son utilité sociale.

C'est d'autant plus indispensable que dans le monde contemporain les technologies numériques créent une convergence frappante d'outils et de méthodes, au-dessus des temps de la vie, au-dessus des métiers, des hiérarchies et des frontières. En faisant du "travail » de ses élèves un objet mieux défini et plus valorisé, en les invitant à un ensemble des démarches individuelles et collectives débouchant sur une "production» véritable, l'école peut aussi trouver les moyens de conduire ses élèves à mieux réfléchir à ce qu'est le travail humain en un sens plus large, à son organisation, ses contraintes, ainsi qu'à sa division sociale.

La plupart des articles qu'on va lire nous convient au fond à ces deux questions : si l'école parvient véritablement à conduire les élèves à une conquête plus assurée de l'autonomie de pensée et d'agir en revisitant les travaux qu'elle leur prescrit, comment faire en sorte que cette autonomie soit effectivement accessible et profitable à tous ? Comment ce frottement à un travail authentique au cœur de l'école peut-il permettre à ses acteurs, élèves compris, de se doter progressivement d'une conscience critique plus vive du rôle que jouent les apprentissages dans la reproduction sociale? 